ISSN: 1808-8759

\title{
ANÁLISE MULTIVARIADA NA DIFERENCIAÇÃO ENTRE MANEJOS DO SOLO CULTIVADO COM CAFÉ
}

\author{
Alessandra Fagioli da Silva ${ }^{1}$, Ana Paula Barbosa ${ }^{2}$, Célia Regina Lopes Zimback ${ }^{3}$, Julião \\ Soares de Souza Lima ${ }^{4} \&$ Paulo Milton Barbosa Landim ${ }^{5}$
}

\begin{abstract}
RESUMO: O objetivo deste trabalho foi identificar as diferenças entre manejos do solo cultivado com café arabica por meio da análise de componentes principais e geoestatística. As amostras de solo foram coletadas em duas áreas comerciais de café arábica sob manejo orgânico e convencional em dois grids georreferenciados. Em cada sistema de manejo foram amostrados 40 pontos, na profundidade de 0-0,10 m, para análises químicas de $\mathrm{pH}$ em água, potássio (K), fósforo $(\mathrm{P})$, cálcio $(\mathrm{Ca})$, magnésio $(\mathrm{Mg})$ e alumínio $(\mathrm{Al})$, carbono orgânico total (COT), carbono solúvel em água (COS) e evolução do $\mathrm{CO}_{2}\left(\mathrm{Ev} . \mathrm{CO}_{2}\right)$. A análise multivariada foi realizada pelo método de componentes principais (ACP) envolvendo as variáveis em estudo. A análise geoestatística foi utilizada na determinação de modelos teóricos de variogramas e na interpolação por krigagem dos dados que foram gerados por meio da aplicação da ACP. No manejo orgânico, os atributos do solo foram mais correlacionados entre si, do que no manejo convencional. Pela análise de componentes principais verificou-se que os atributos com maior peso fatorial na componente 1 foram pH e saturação por bases e, matéria orgânica e carbono solúvel em água na componente 2, para ambos os manejos..
\end{abstract}

PALAVRAS-CHAVE: Manejo orgânico, análise de componentes principais, krigagem.

\section{DIFFERENCES BETWEEN TWO SOIL MANAGEMENT SYSTEMS OF ARABICA COFFEE PLANTATION}

ABSTRACT: The goal of this study was to identify the differences between two soil management systems of arabica coffee plantations using geostatistics. Soil samples were collected in two coffee plantations under organic and conventional management systems in two georeferenced grid. For chemical analysis of $\mathrm{pH}$, potassium $(\mathrm{K})$, phosphorus $(\mathrm{P})$, calcium $(\mathrm{Ca})$, magnesium $(\mathrm{Mg})$ and aluminum $(\mathrm{Al})$, organic carbon total (OCT), water-soluble carbon (WSC) and $\mathrm{CO}_{2}$ evolution $\left(\mathrm{CO}_{2}\right.$ Ev. $)$, data were collected for each management system in 40 points, in the depth of $0-0.10$ meters,. The multivariate analysis was performed with the method of principal components analysis (PCA) involving all the variables under study. The geostatistical analysis was applied to determine the theoretical models of variograms and data interpolation, generated by PCA. In the organic management, the attributes are better correlated than in the conventional management. For both managements, the attributes with the highest weight factor in component 1 are $\mathrm{pH}$, base saturation, and organic matter and in component 2, water soluble.

KEYWORDS: organic management, principal component analysis, kriging.

\footnotetext{
${ }^{1}$ e ${ }^{2}$ Faculdade de Ciências Agronômicas da Universidade Estadual Paulista - FCA/UNESP, Departamento de Recursos Naturais- Ciências do solo.E-mail: alefagioli@hotmail.com; anap_barbosa@yahoo.com.br ${ }^{3}$ Orientadora e docente da Faculdade de Ciências Agronômicas da Universidade Estadual Paulista - FCA/UNESP, Departamento de Recursos Naturais- Ciências do solo. E-mail: czimback@gmail.com
}

\footnotetext{
${ }^{4}$ Centro de Ciências Agrárias da Universidade Federal do Espirito Santo - CCA/UFES, Departamento de Engenharia Rural. E-mail: limajss@yahoo.com.br

${ }^{5}$ Instituto de Geociências e Ciências Exatas da Universidade Estadual Paulista - IGCE/UNESP. E-mail: plandim@rc.unesp.br
} 


\section{INTRODUÇÃO}

O Brasil é o país que apresenta a maior produção mundial de café convencional, sendo também o maior exportador, gerando uma receita anual de aproximadamente 3 bilhões de dólares (EMBRAPA, 2007).

Em relação à produção de café orgânico, observa-se que esta tem aumentado a cada ano no Brasil, sendo um reflexo da demanda mundial por alimentos orgânicos, considerados de qualidade e saudáveis (LUCIA et al., 2009). O conjunto dos processos de produção da agricultura orgânica é baseado na fertilidade do solo, como função direta do seu conteúdo de matéria orgânica (ORMOND et al., 2002).

As correlações entre as propriedades do solo e sua extensão na variabilidade espacial, levaram cientistas do solo a utilizar a combinação de técnicas de análise multivariada e geoestatística (GOMES et al., 2004; VALLADARES et al., 2008; SILVA; LIMA, 2012), na tentativa de reduzir a dimensionalidade elevada dos dados coletados e extrair as variáveis mais significativas ou uma combinação adequada das mesmas. A identificação de um conjunto reduzido de variável altamente informativo, não serve apenas para redução de custos em projetos de mapeamento de solos, mas também oferece a oportunidade para revelar sequências de processos pedogenéticos e elucidar o papel dos parâmetros do solo (THEOCHAROPOULOS et al., 1997).

A técnica multivariada, conhecida como análise de componentes principais (ACP), é uma ferramenta estatística, que permite estabelecer não só a identificação de diferenças entre tratamentos, mas também qual é o atributo que mais contribui para a separação entre as áreas estudadas, permitindo, assim, a eliminação de tempo e dinheiro gastos com aqueles atributos do solo que apresentam pouca informação da fertilidade do solo (CRUZ-CASTILHO et al., 1994; BARETTA et al., 2007).

Essa abordagem é muito útil porque, além de identificar os atributos químicos ou microbianos do solo mais relevantes para a separação das áreas, permite considerálo como um indicador sensível para avaliação da intervenção antrópica (CHOROMANSKA; DELUCA, 2001; FRANCHINI et al., 2007).

Atualmente são conhecidos efeitos isolados de diferentes manejos e uso do solo nos atributos físicos, químicos e biológicos. No entanto, há a necessidade de avaliar esses atributos em conjunto. Assim, a utilização de análise multivariada dos dados poderá indicar diferenças e estabelecer as relações entre os tratamentos e os atributos avaliados, tornando-se uma ferramenta importante na análise das informações obtidas (CRUZ-CASTILHO et al., 1994).

A análise estatística multivariada fornece, portanto, técnicas para classificar a relação entre os atributos do solo. Porém, abordagens de estatística multivariada clássica ignoram a correlação espacial entre os pontos de amostragem (KORRE, 1999; LIN, 2002). Os métodos de geoestatística multivariada juntam correlações espaciais e relacionamento multivariado para detectar e mapear as diferentes fontes de variação espacial (MONTANARI et al., 2012).

O objetivo deste trabalho foi identificar as diferenças entre os manejos do solo cultivado com café arabica por meio da análise de componentes principais (ACP) e geoestatística.

\section{MATERIAL E MÉTODOS}

As amostras de solo foram coletadas em duas áreas comerciais de café arábica (Coffea arabica cv.catuaí 44) sob dois tipos de manejo do solo: orgânico e convencional, no município de Irupi, região do entorno do Caparaó, sul do Estado do Espírito Santo. A região situa-se na latitude $20^{\circ} 20^{\prime} 43^{\prime \prime}$ S e longitude $41^{\circ} 38^{\prime}$ 28" W com altitude média de $730 \mathrm{~m}$ e temperatura média anual de $20^{\circ} \mathrm{C}$. O solo representativo das áreas de estudo é classificado como Latossolo Vermelho-amarelo (EMBRAPA, 2006) com declividade suave ondulado.

O sistema orgânico de manejo do cafeeiro deste trabalho iniciou-se no processo de transição de convencional para orgânico, no ano de 1998, é manejado organicamente, e possui selo de qualidade orgânica Chão Vivo, constituindo um sistema orgânico consolidado. O espaçamento da cultura é de 2,0 x 1,0 m, com plantio em nível. As plantas espontâneas que ocorrem nas entrelinhas do cafeeiro são: capim-marmelada (Brachiaria plantaginea (Link) Hitchc.), picão (Bidens pilosa L.) e trapoeraba (Commelina benghalensis L.), que são controladas com uso de roçadora costal e uma leve capina manual. Nas entrelinhas foram plantadas leguminosas, nas quantidades de 150, 60 e $80 \mathrm{~kg}$ de sementes, por hectare, de feijão de porco (Canavalia ensiformis (L.) DC.), mucuna-preta (Mucuna pruriens utilis (Wall. ex Wight)L.H.Bailey) e mucuna-anã ((Mucuna pruriens utilis (Wall. Ex Wight)L.H.Bailey)), respectivamente, em sistemas de rotação, conforme a orientação da certificadora. Quanto à adubação, uma vez por ano, são aplicados 8 kg de composto orgânico por cova, com a seguinte composição: 4235,97 $\mathrm{mg} \mathrm{dm}^{-3} \mathrm{de}$ $\mathrm{K} ; 207,79 \mathrm{mg} \mathrm{dm}^{-3}$ de P; 46,50 $\mathrm{cmol}_{\mathrm{C}} \mathrm{dm}^{-3}$ de Ca; 27 $\mathrm{cmol}_{\mathrm{c}} \mathrm{dm}^{-3}$ de $\mathrm{Mg}$, conforme comunicado pelo proprietário. No período de chuvas é aplicado, mensalmente, na forma de pulverização foliar, o fertilizante de nome comercial Humato de Macota, cujo rótulo indica ser um produto de $\mathrm{pH}$ neutro, constituído por ácido húmico $\left(178,35 \mathrm{mg} \mathrm{L}^{-1}\right)$, ácido fúlvico $(143,83$ $\left.\mathrm{mg} \mathrm{L}^{-1}\right)$, carbono $\left(3,09 \mathrm{mg} \mathrm{L}^{-1}\right)$, cálcio $\left(250 \mathrm{mg} \mathrm{L}^{-1}\right)$, magnésio $\left(176,90 \mathrm{mg} \mathrm{L}^{-1}\right)$, potássio $\left(25 \mathrm{mg} \mathrm{L}^{-1}\right)$, nitrogênio $\left(54,20 \mathrm{mg} \mathrm{L}^{-1}\right)$, na proporção de $700 \mathrm{~mL}$ do produto por 20 litros de água, utilizando volume de aplicação de 200 a $220 \mathrm{~L} \mathrm{ha}^{-1}$.

A área de café convencional tem espaçamento de 3,0 x $1,5 \mathrm{~m}$. As plantas espontâneas que ocorrem nas entrelinhas são: capim-marmelada (B. plantaginea), picão (B. pilosa) e trapoeraba (C. benghalensis), controladas com o uso de herbicida comercial Roundup ${ }^{\circledR}$ (glyphosate), na dose de $1,0 \mathrm{~L} \mathrm{ha}^{-1}$, e por meio de 
capinas manuais, mantendo-se baixa cobertura no solo. Na adubação é utilizado o formulado NPK, conforme o manual de recomendação de calagem e adubação para o estado do Espírito Santo (DADALTO; FULLIN, 2001).

A amostragem do solo foi realizada com espaçamento irregulares entre locais de amostras por meio de um grid georreferenciado (Figura 1). Em cada sistema de manejo foram amostrados 40 pontos, na profundidade de 0-0,10 $\mathrm{m}$, na projeção da copa do cafeeiro, para a realização das análises químicas: $\mathrm{pH}$, potássio (K), fósforo (P), cálcio
(Ca), magnésio (Mg), alumínio (Al), capacidade de troca de cátions (CTC) e saturação por bases (V\%) (EMBRAPA, 1997). As análises de carbono orgânico total (COT), carbono solúvel em água (COS), foram realizadas de acordo com a metodologia descrita por PASSOS (2000) e as de evolução do $\mathrm{CO}_{2}\left(\mathrm{Ev} . \mathrm{CO}_{2}\right)$, conforme MENDONÇA E MATOS (2005).

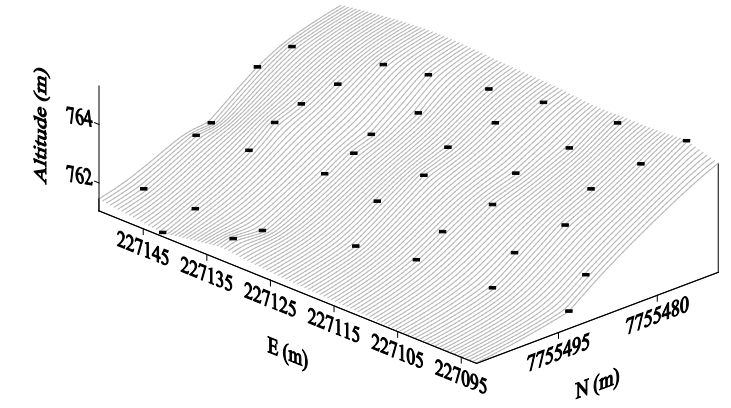

(a)

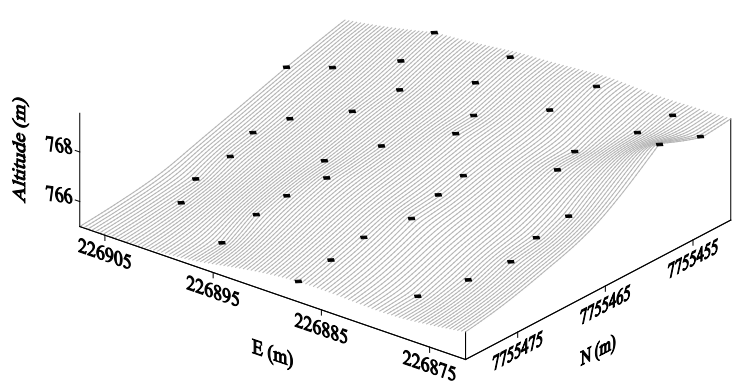

(b)

Figura 1 - Malha de pontos amostrais nas áreas de manejo orgânico (a) e convencional (b) do solo cultivado com café.

Para a aplicação da análise multivariada os dados foram padronizados, pois apresentavam grandezas diferentes, e em seguida foi realizada a análise de correlação de Pearson (n-1) com probabilidade $\mathrm{p}>0,05$.

A análise de componentes principais (ACP), que consiste numa transformação linear de " $m$ ” variáveis originais em “m” novas variáveis (componentes), de tal modo que a primeira nova variável (componente 1) computada seja responsável pela maior variação possível existente no conjunto de dados, a segunda (componente 2) pela maior variação possível restante, e assim por diante até que toda a variação do conjunto tenha sido explicada, foi realizada envolvendo todas as variáveis em estudo, a partir da qual foi reduzido o conjunto de dados em combinações lineares, ditos componentes principais para identificação daqueles que expliquem a maior parte da variabilidade existente nos sistemas de manejo do solo.

A análise multivariada foi realizada no programa XLStat e a análise geoestatística no $\mathrm{GS}^{+} 9.0$ (ROBERTSON, 2008).

A análise geoestatística foi utilizada na determinação de modelos teóricos de variogramas e interpolações por krigagem da contribuição das observações (\%), que foram gerados pela aplicação da ACP. A seleção do variograma teórico foi feita com base na menor soma dos quadrados dos resíduos e do maior coeficiente de correlação na validação cruzada. A estimação dos valores em locais não medidos foi realizada por krigagem ordinária para confecção dos mapas das componentes principais.

\section{RESULTADOS E DISCUSSÃO}

As correlações lineares entre as variáveis do solo cultivado com café sob manejo orgânico e convencional encontram-se na figura 2.

Observa-se que no manejo orgânico, a maior correlação $(0,973)$ foi entre o $\mathrm{pH}$ e $\mathrm{V}(\%)$, sendo que o $\mathrm{pH}$ e $\mathrm{Ca}, \mathrm{pH}$ e Evolução do CO2, Ca e V (\%), Ca e Evolução do CO2, CTC e V (\%) e V (\%) e Evolução do CO2 apresentaram correlações maiores que 0,8 , e o pH e CTC apresentaram correlação de $-0,812$. 

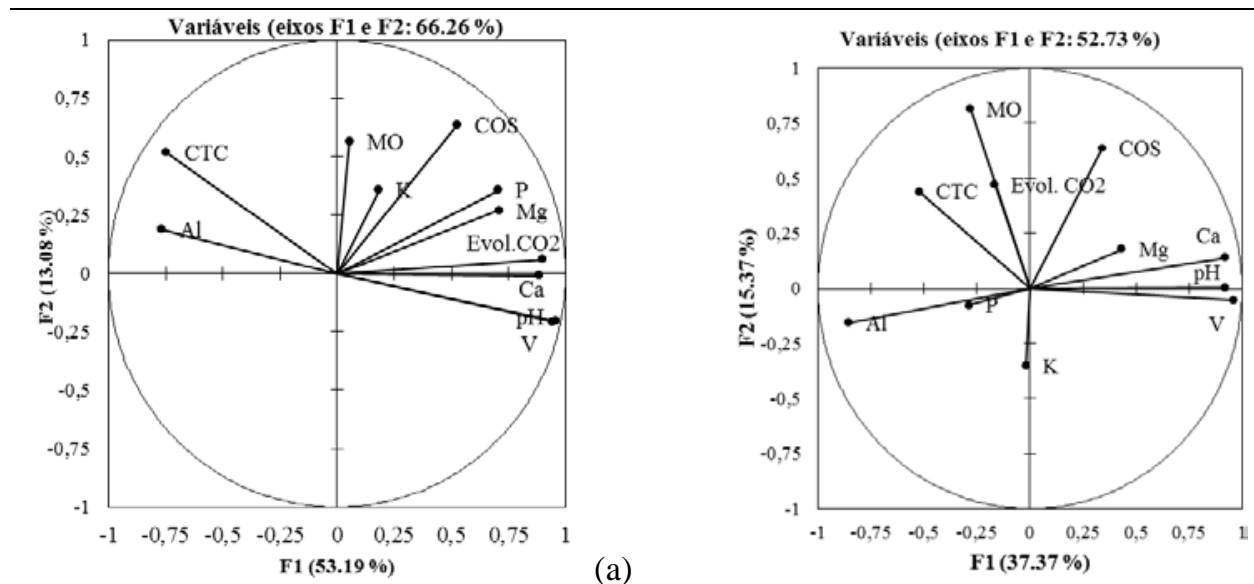

(b)

Figura 2 - Correlações dos atributos do solo $\mathrm{pH}$ : $\mathrm{pH}$ em áqua; $K$ : potássio ( $\left.m g \mathrm{dm}^{-3}\right)$; $P$ : fósforo $\left(\mathrm{mg} \mathrm{dm}^{-3}\right)$; Ca: cálcio $\left(\mathrm{cmol}_{c} \mathrm{dm}^{-3}\right) ; \mathrm{Mg}$ : magnésio $\left(\mathrm{cmol}_{c} \mathrm{dm}^{-3}\right) ; \mathrm{Al}$ : alumínio $\left(\mathrm{cmol}_{c} \mathrm{dm}^{-3}\right) ; \mathrm{CTC}$ : capacidade de troca de cations $\left(\mathrm{cmol}_{c} \mathrm{dm}^{-3}\right)$; $\mathrm{V}$ : saturação por bases (\%); MO: matéria orgânica $\left(g \mathrm{~kg}^{-1}\right)$; COS: carbono solúvel em água ( $\left.\mathrm{mg} \mathrm{kg}^{-1}\right)$; e Evol. $\mathrm{CO}_{2}$ : evolução do $\mathrm{CO}_{2}\left(\mathrm{~g} \mathrm{~kg}^{-1}\right)$ cultivado com café sob manejo orgânico (a) e convencional (b).

No manejo convencional, nota-se que a maior correlação $(0,921)$ foi entre o $\mathrm{pH}$ e Ca, sendo que o $\mathrm{pH}$ e $\mathrm{V}(\%)$ e $\mathrm{Ca}$ e V (\%) apresentaram correlações positivas maiores que 0,8 .

ALBUQUERQUE et al. (2005) encontraram alta correlação entre $\mathrm{pH}$ e Ca, e pH e V (\%), em manejo convencional do solo, concordando com os dados encontrados neste trabalho. As altas correlações entre $\mathrm{pH}, \mathrm{Ca}, \mathrm{Mg}$ e V\% são esperadas uma vez que quando se adiciona calcário no solo, para correção da acidez, Estão sendo ao mesmo tempo adicionados Ca e $\mathrm{Mg}$, os quais influenciam no valor da V\%

Em ambos os manejos (orgânico e convencional), o atributo $\mathrm{K}$ foi o que apresentou menor correlação. Em manejo convencional, ALBUQUERQUE et al. (2005) e MONTEZANO et al. (2006) encontraram baixa correlação de $\mathrm{K}$ com os demais atributos. A baixa correlação entre o K e os demais atributos do solo ocorre pelo fato do K ser móvel no solo, podendo ser absorvido rapidamente pelas plantas e podendo ser perdido por lixiviação e por oxidação quando em contato com o oxigênio do ar.

Embora a variável K tenha apresentado baixa correlação com os demais atributos do solo, segundo SILVA et al. (2002), para obtenção de altas produtividades e grãos de café de qualidade superior, é necessária, além de outras práticas, uma recomendação adequada de fertilização potássica.

Na Tabela 1 estão apresentadas as porcentagens de variância total que são explicadas pelas componentes $1 \mathrm{e}$ 2, em ambos os manejos, sendo que as informações mais relevantes dos dados amostrais originais estão contidas nessas duas componentes principais.
Tabela 1 - Resultado da análise de componentes principais para o manejo orgânico e convencional do solo cultivado com café.

\begin{tabular}{lcccc}
\hline & MOF1 & MOF2 & MCF1 & MCF2 \\
\hline Autovalor & 5,850 & 1,438 & 4,110 & 1,690 \\
$\begin{array}{l}\text { Variabilidade } \\
\text { (\%) }\end{array}$ & 53,185 & 13,076 & 37,367 & 15,366 \\
\% acumulada & 53,185 & 66,261 & 37,367 & 52,733 \\
\hline
\end{tabular}

MOF1: componente principal 1 do manejo orgânico; MOF2: componente principal 2 do manejo orgânico; MCF1: componente principal 1 do manejo convencional; MCF2: componente principal 2 do manejo convencional.

Segundo MINGOTI (2005), normalmente, é utilizado as componentes principais que representam mais que $80 \%$ da variabilidade acumulada, no entanto, para que as componentes possam ser utilizadas adequadamente, o mais indicado é que contemplem as variáveis com maior influência nas outras variáveis.

$\mathrm{Na}$ análise de componentes principais, o $\mathrm{V}$ (\%) foi o atributo que apresentou maior carga fatorial na componente 1 em ambos os manejos, seguido pelos atributos pH e cálcio (Tabela 2). No manejo orgânico, o atributo evolução do $\mathrm{CO}_{2}$ foi o que mais contribuiu na componente 1, enquanto no manejo convencional contribuiu pouco, mostrando que no manejo orgânico do solo a atividade microbiana tem influência na ciclagem dos nutrientes para as plantas, proporcionando um solo mais fértil.

THEODORO et al. (2003) verificaram que o manejo do café orgânico registrou maior alteração nas características químicas do solo (na profundidade de 0 - 
0,20 m) em relação ao convencional, apresentando incrementos no $\mathrm{pH}$ e nos valores de $\mathrm{Ca}, \mathrm{Mg}, \mathrm{K}, \mathrm{P}, \mathrm{Zn}, \mathrm{B}$, CTC do solo, SB, V\% e diminuição do Al trocável.

Tabela 2 - Contribuições dos atributos (\%) do solo cultivado com café sob diferentes manejos em relação às componentes principais.

\begin{tabular}{lrrrr}
\hline \multicolumn{1}{c}{ Atributos } & \multicolumn{1}{c}{ MOF1 } & \multicolumn{1}{c}{ MOF2 } & \multicolumn{1}{c}{ MCF1 } & \multicolumn{1}{c}{ MCF2 } \\
\hline $\mathrm{pH}$ & 15,146 & 2,922 & 20,524 & 0,001 \\
$\mathrm{~K}$ & 0,571 & 8,847 & 0,008 & 7,316 \\
$\mathrm{P}$ & 8,511 & 8,887 & 2,026 & 0,353 \\
$\mathrm{Ca}$ & 13,304 & 0,007 & 20,623 & 1,154 \\
$\mathrm{Mg}$ & 8,569 & 5,036 & 4,566 & 1,886 \\
$\mathrm{Al}$ & 10,042 & 2,461 & 17,835 & 1,431 \\
$\mathrm{CTC}$ & 9,543 & 18,517 & 6,653 & 11,417 \\
$\mathrm{~V}$ & 15,687 & 2,857 & 22,367 & 0,163 \\
Evol.CO & 13,882 & 0,251 & 0,679 & 13,091 \\
$\mathrm{MO}$ & 0,052 & 22,218 & 1,921 & 39,225 \\
$\mathrm{COS}$ & 4,693 & 27,997 & 2,798 & 23,963 \\
\hline
\end{tabular}

MOF1: componente principal 1 do manejo orgânico; MOF2: componente principal 2 do manejo orgânico; MCF1: componente principal 1 do manejo convencional; MCF2: componente principal 2 do manejo convencional; $\mathrm{pH}$ : $\mathrm{pH}$ em água; $\mathrm{K}$ : potássio $\left(\mathrm{mg} \mathrm{dm}^{-3}\right)$; $\mathrm{P}$ : fósforo $\left(\mathrm{mg} \mathrm{dm}^{-3}\right)$; Ca: cálcio (cmolc $\mathrm{dm}^{-3}$ ); Mg: magnésio (cmolc $\mathrm{dm}^{-3}$ ); Al: alumínio $\left(\mathrm{cmolc} \mathrm{dm}^{-3}\right.$ ); CTC: capacidade de troca de cations (cmolc dm${ }^{-}$ 3); V: saturação por bases (\%); MO: matéria orgânica $\left(\mathrm{g} \mathrm{kg}^{-1}\right)$; COS: carbono solúvel em água ( $\left.\mathrm{mg} \mathrm{kg}^{-1}\right)$; e Evol. $\mathrm{CO}_{2}$ : evolução do $\mathrm{CO}_{2}\left(\mathrm{~g} \mathrm{~kg}^{-1}\right)$.
Os atributos de maior contribuição no componente principal 2 são aqueles relacionados à matéria orgânica e sua solubilidade (MO e COS), independente do sistema de manejo utilizado. Dentre os benefícios gerados pela MO, destacam-se a melhoria das condições físicas do solo e o fornecimento de energia para o crescimento microbiano (SILVA; RESCK, 1997), o que reflete em maior ciclagem de nutrientes e aumento da CTC do solo (PAES et al., 1996).

A distribuição espacial dos pontos amostrais em relação ao teores dos atributos do solo cultivado com café sob manejo orgânico e convencional está representado na figura 3.

Na área de manejo orgânico a maioria dos pontos amostrais está associada a valores positivos da componente principal 1 e valores negativos do eixo da componente principal 2, apresentando os maiores valores de $\mathrm{pH}$ e $\mathrm{V}$ (\%) e, consequentemente, os menores valores de Al.

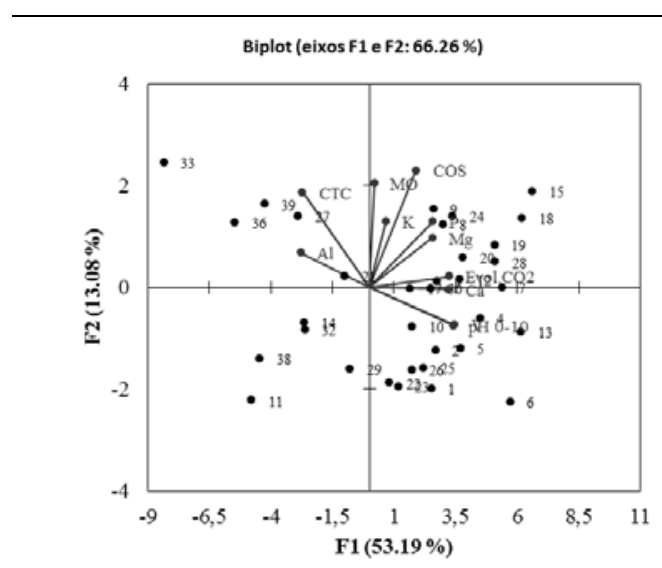

(a)

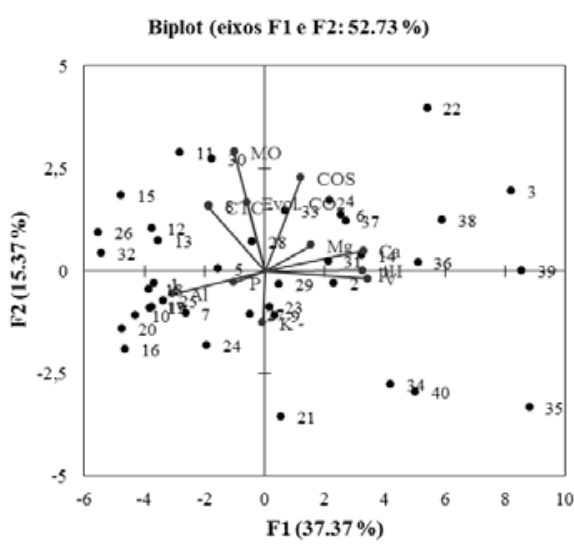

(b)

Figura 3 - Correlações e distribuição dos pontos amostrais em relação aos teores dos atributos do solo pH: pH em água; K: potássio $\left(\mathrm{mg} \mathrm{dm}^{-3}\right) ; \mathrm{P}$ : fósforo $\left(\mathrm{mg} \mathrm{dm}^{-3}\right) ; \mathrm{Ca}$ : cálcio $\left(\mathrm{cmol}_{\mathrm{c}} \mathrm{dm}^{-3}\right) ; \mathrm{Mg}$ : magnésio $\left(\mathrm{cmol}_{c} \mathrm{dm}^{-3}\right)$; Al: alumínio $\left(\mathrm{cmol}_{c} \mathrm{dm}^{-3}\right)$; CTC: capacidade de troca de cations $\left(\mathrm{cmol}_{c} \mathrm{dm}^{-3}\right) ; \mathrm{V}$ : saturação por bases (\%); MO: matéria orgânica $\left(\mathrm{g} \mathrm{kg}^{-1}\right)$; COS: carbono solúvel em água $\left(\mathrm{mg} \mathrm{kg}^{-1}\right)$; e Evol. $\mathrm{CO}_{2}$ : evolução do $\mathrm{CO}_{2}\left(\mathrm{~g} \mathrm{~kg}^{-1}\right)$ cultivado com café sob manejo orgânico (a) e convencional (b).

Já, para o manejo convencional, observou-se que a maioria dos pontos amostrais estão associados a valores negativos da componente principal 1 e com valores negativos do eixo da componente principal 2, com os maiores valores de $\mathrm{Al}$ e, consequentemente, os menores valores de $\mathrm{pH}$ e V (\%).
Os atributos químicos e biológicos do solo (Figura 3) em sistemas orgânicos apresentam-se mais correlacionados do que no sistema convencional, mostrando que o manejo orgânico do solo tem mais atributos para disponibilizar para a nutrição do cafeeiro, aumentando a qualidade do sistema orgânico. 
Ao realizar a análise variográfica das componentes 1 e 2 das áreas de manejo orgânico e convencional, verificouse que apenas a componente 1 do manejo convencional apresentou correlação espacial, ajustando-se ao modelo esférico (Figura 4), com alcance de 10,20 m e com forte dependência espacial (IDE $\geq 75 \%$ ), conforme ZIMBACK (2001).

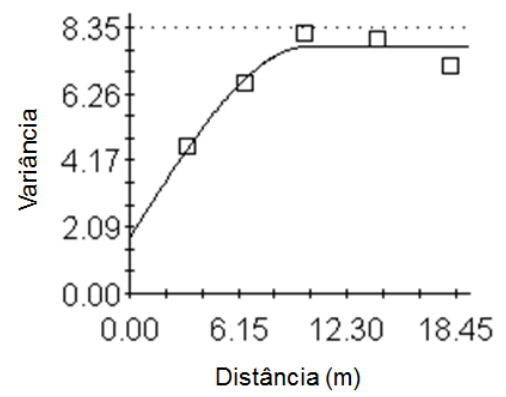

Figura 4 - Variograma da componente principal 1 da área cultivada com café sob manejo convencional.

Foi possível estimar pela krigagem valores em locais não amostrados (Figura 5), devido ao bom ajuste do variograma, que foi confirmado pela validação cruzada.

Observa-se no mapa de krigagem que a região de maior fertilidade do solo está representada pelos tons escuros, com maiores teores de $\mathrm{Ca}, \mathrm{pH}$ e $\mathrm{V}(\%)$ e a região clara representa teores mais elevados de $\mathrm{Al}$, sendo que as regiões de maiores concentração dos nutrientes são as áreas receptoras do fluxo de água, de acordo com a topografia do terreno, o que ocorre devido a baixa cobertura do solo por material orgânico.

Em solo com maior cobertura vegetal a água da chuva possui maior infiltração do que em solos com baixa cobertura vegetal.

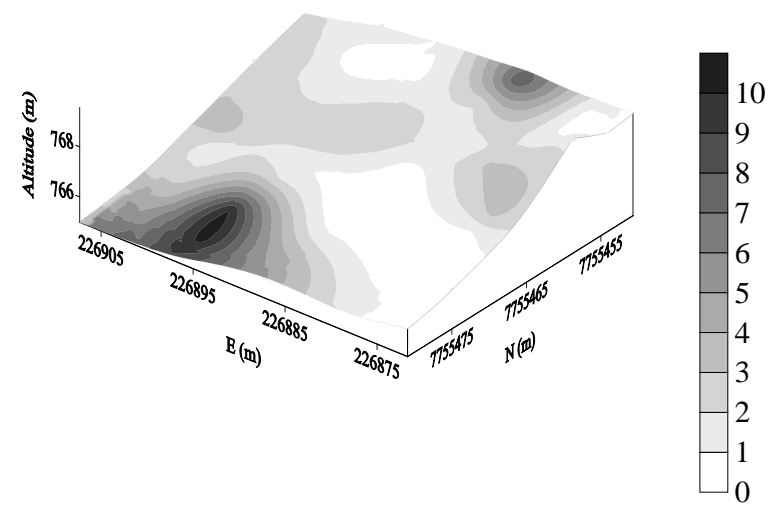

Figura 5 - Mapa da distribuição espacial da componente principal 1 da área cultivada com café sob manejo convencional.
Com estes resultados pode-se definir zonas de manejo, considerando o conjunto de atributos explicados pela componente 1 .

\section{CONCLUSÃO}

Ao verificar as diferenças entre manejos do solo cultivado com café arabica observa-se que no manejo orgânico os atributos do solo são mais correlacionados entre si, do que no manejo convencional, com isso há maior disponibilidade de nutrientes para as plantas no manejo orgânico.

Pela comparação dos dois manejos verifica-se que no manejo orgânico, a evolução do $\mathrm{CO} 2$ (atividade microbiana) tem maior influência na ciclagem dos nutrientes pelas plantas que no manejo convencional.

\section{AGRADECIMENTO}

Ao Grupo de Estudos e Pesquisas Agrárias Georreferenciadas (GEPAG). À Capes pela concessão de bolsa ao primeiro e segundo autor.

\section{REFERÊNCIAS}

ALBUQUERQUE, J. A. et al. Relação de atributos do solo com a agregação de um latossolo vermelho sob sistemas de preparo e plantas de verão para cobertura do solo. Revista Brasileira de Ciência do Solo, Viçosa, v. 29, p. 415-424, 2005.

\section{BARETTA, D. Fauna do solo e outros atributos edáficos como indicadores da qualidade ambiental em áreas com Araucaria Angustifolia no Estado de São Paulo. 2007. 158 f. Tese (Doutorado em Agronomia)-Escola Superior de Agricultura Luiz de Queiroz, Universidade do Estado de São Paulo, Piracicaba, 2007.}

CHOROMANSKA, U.; DELUCA, T. H. Prescribed fire alters the impact of wildfire on soil biochemical properties in a ponderosa pine forest. Soil Science Society of America Journal, Madison, v. 65, p. 232238, 2001.

CRUZ-CASTILHO, J. G. et al. Applications of canonical discriminate analysis in horticultural research. Horticultural Science, Praha, v. 29, p. 1115-1119, 1994.

DADALTO, G. G.; FULLIN, E. A. Manual de recomendação de calagem e adubação para o estado do Espírito Santo: $4^{\mathrm{a}}$ aproximação. Vitória: SEEA/INCAPER, 2001. 266 p.

EMBRAPA. Centro Nacional de Pesquisa de Solos. Manual de métodos de análise de solo. 2. ed. Rio de Janeiro: Embrapa, 1997. 212 p. 
EMBRAPA. Centro Nacional de Pesquisa de Solos. Sistema brasileiro de classificação de solos. 2.ed. Rio de Janeiro: Embrapa, 2006. 374 p.

EMBRAPA. Café convencional. 2007. Disponível em: <http://www.embrapa.br>. Acesso em: 20 jan. 2007.

FRANCHINI, J. C. et al. Microbiological parameters as indicators of soil quality under various soil management and crop rotation systems in Southern Brazil. Soil and Tillage Research, New York, v. 92, p. 18-29, 2007.

GOMES, J. B. V. et al. Análise de componentes principais de atributos físicos, químicos e mineralógicos de solos do bioma cerrado. Revista Brasileira de Ciências do Solo, Viçosa, v. 28, p. 137-153, 2004.

KORRE, A. Statistical and spatial assessment of soil heavy metal contamination in areas of poorly recorded, complex sources of pollution. Stochastic

Environmental Research and Risk Assessment, New York, v. 13, p. 288-316, 1999.

LIN, Y. P. Multivariate geostatistical methods to identify and map spatial variations of soil heavy metals.

Environmental Geology, Berlin, v. 42, p. 1-10, 2002.

LUCIA, S. M. D. et al. Características visuais da embalagem de café no processo de decisão de compra pelo consumidor. Ciência e agrotecnologia, Lavras, v. 33, p. 1758 -1764, 2009. Edição Especial.

MENDONÇA, E. S.; MATOS, E. S. Matéria orgânica do solo: métodos de análises. Ponte Nova: D\&M Gráfica e Editora , 2005. 107 p.

MINGOTI, S. A. Análise de dados através de métodos de estatística multivariada. Belo Horizonte. Editora UFMG, 2005. 295 p.

MONTANARI, R. et al. The use of scaled semivariograms to plan soil sampling in sugarcane fields. Precision Agriculture, Genebra, v. 35, p. 1-11, 2012.

MONTEZANO, Z .F.; CORAZZA, E. J.; MURAOKA, T. Variabilidade espacial da fertilidade do solo em área cultivada e manejada homogeneamente. Revista Brasileira de Ciências do Solo, Viçosa, v. 30, p. 839847, 2006.

ORMOND, J. G. P. et al. Agricultura orgânica: quando o passado é futuro. Rio de Janeiro: BNDES, 2002. 35 p.

PAES, J. M. V. et al. Decomposição da palha de café em três tipos de solo e sua influência sobre a CTC e o pH. Revista Ceres, Viçosa, v. 43, p. 337-392, 1996.

PASSOS, R. R. Carbono orgânico e nitrogênio em agregados de um Latossolo vermelho sob duas coberturas vegetais. 2000. 115 f. Tese (Doutorado em
Agronomia)-Universidade Federal de Viçosa, Viçosa, 2000.

ROBERTSON, G. P. GS ${ }^{+}$: geoestatistics for the environmental sciences. GS+ user's guide version 9.0. Plainwell: Gamma Desing Software, 2008. 152 p.

SILVA, E. B.; NOGUEIRA, F. D.; GUIMARÃES P. T. G. Qualidade dos grãos de café em função de doses de potássio. Acta Scientiarum, Maringá, v. 24, p. 12911297, 2002.

SILVA, J. E.; RESCK, D. V. S. Matéria orgânica do solo. In: VARGAS, M. A. T.; HUNGRIA, M. (Ed.). Biologia dos solos dos cerrados. Planaltina: Embrapa, CPAC, 1997. p. 467-524.

SILVA, S. A.; LIMA, J. S. S. Avaliação da variabilidade do estado nutricional e produtividade de café por meio da análise de componentes principais e geoestatística. Revista Ceres, Viçosa, v. 59, p. 271-277, 2012.

THEOCHAROPOULOS, S. P.; PETRAKIS, P. V.; TRIKATSOULA, A. Multivariate analysis of soil grid data as a soil classification and mapping tool: the case study of a homogeneous plain in Vagia, Viotia, Greece. Geoderma, Amsterdam, v. 7, p.63-79, 1997.

THEODORO, V. C. A. et al. Alterações químicas em solo submetido a diferentes formas de manejo do cafeeiro. Revista Brasileira de Ciência do Solo, Viçosa, v. 27, p. 1039-1047, 2003.

VALLADARES, G. S. et al. Análise dos componentes principais e métodos multicritério ordinais no estudo de organossolos e solos afins. Revista Brasileira de Ciência do Solo, Viçosa, v. 32, p. 285-296, 2008.

ZIMBACK, C. R. L. Análise espacial de atributos químicos de solos para fins de mapeamento da fertilidade do solo. 2001. 114 f. Tese (Livre-Docência) Faculdade de Ciências Agronômicas, Universidade Estadual Paulista. Botucatu. 